\title{
Experiências de enfermeiras da atenção primária à saúde no atendimento pré-natal de
} adolescentes

\section{Experiences of primary health care nurses in the prenatal care of adolescents}

\section{Experiencias de enfermeras de la atención primaria a la salud en el atendimiento pre- natal de adolescentes}

\section{Recebido: 07/06/2017 \\ Aprovado: 20/11/2017 \\ Publicado: 30/03/2018}

\section{Patricia Wottrich Parenti ${ }^{1}$ Lucia Cristina Florentino Pereira da Silva² Evelyn Priscila Santinon Sola ${ }^{3}$ Kelly Cristina Pereira Máxima Venâncio ${ }^{4}$ Fernanda Marçal Ferreira ${ }^{5}$ Joyce da Costa Silveira de Camargo ${ }^{6}$}

Este estudo tem como objetivo conhecer as experiências de enfermeiras da Atenção Primária de Saúde (APS) na assistência pré-natal de adolescentes. Tratou-se de uma pesquisa qualitativa usando entrevistas semiestruturadas com 19 enfermeiras atuantes na APS no atendimento pré-natal de adolescentes, analisadas segundo a Análise de Conteúdo Temática de Bardin. Foram identificadas três subcategorias pertencentes à categoria "Atendimento de pré-natal": Atenção pré-natal às adolescentes; Enfoque de risco biológico versus risco social; e, Aspectos prioritários para assistência pré-natal. A adolescente não recebe atenção pré-natal específica e direcionada. Há necessidade de mais tempo de consulta, incluindo: acolhimento diferenciado, estimulo a criação do vínculo e de melhoria da qualidade das informações às adolescentes, bem como, medidas para evitar o abandono do pré-natal. As dificuldades citadas foram a adesão ao pré-natal, a falta de diálogo profissional-adolescente, e as situações vividas por essas adolescentes, além das dificuldades sociais.

Descritores: Cuidado Pré-Natal; Gravidez na adolescência; Atenção primária à saúde; Cuidados de enfermagem.

This study aims to know the experiences of primary health care nurses (PHC) in the prenatal care of adolescents. This was a qualitative research using semi-structured interviews with 19 nurses working in PHC in the prenatal care of adolescents, analyzed according to the Analysis of Thematic Content of Bardin. Three subcategories belonging to the category "Prenatal care" were identified: Prenatal care for adolescents; Biological risk versus social risk approach; and, Priority aspects of prenatal care. The adolescent does not receive specific and directed prenatal care. There is a need for more consultation time, including differentiated reception, encouragement of the creation of the link and improvement of the quality of information to adolescents, as well as measures to prevent prenatal abandonment. The difficulties cited were prenatal adherence, lack of professional-adolescent dialogue, and the situations experienced by these adolescents, besides to social difficulties.

Descriptors: Prenatal care; Pregnancy in adolescence; Primary health care; Nursing care.

Este estudio tiene como objetivo conocer las experiencias de enfermeras de la Atención Primaria de Salud (APS) en la asistencia pre-natal de adolescentes. Se trató de una investigación cualitativa usando entrevistas semiestructuradas con 19 enfermeras actuantes en la APS en el atendimiento pre-natal de adolescentes, analizadas según el Análisis de Contenido Temático de Bardin. Fueron identificadas tres subcategorías pertenecientes a la categoría "Atendimiento de pre-natal"; Atención pre-natal a las adolescentes; Enfoque de riesgo biológico versus riesgo social; y, Aspectos prioritarios para asistencia pre-natal. La adolescente no recibe atención pre-natal específica y direccionada. Hay necesidad de más tiempo de consulta, incluyendo: acogida diferenciada, estimula la creación de vínculo y de mejoría de la calidad de las informaciones a las adolescentes, así como, medidas para evitar el abandono del pre-natal. Las dificultades citadas fueron la adhesión al pre-natal, la falta de diálogo profesional-adolescente, y las situaciones vividas por esas adolescentes, además de las dificultades sociales.

Descriptores: Atención prenatal, Embarazo en adolescencia, Atención primaria de salud, Atención de Enfermería.

1. Enfermeira. Doutora em Enfermagem. Docente da Escola de Artes, Ciências e Humanidades da Universidade de São Paulo, São Paulo (SP), Brasil. ORCID: 0000-0001-9321-7169 E-mail: pwparenti@usp.br

2. Enfermeira. Doutora em Enfermagem. Docente da Escola de Artes, Ciências e Humanidades da Universidade de São Paulo, São Paulo (SP), Brasil. ORCID: 0000-0002-7563-6631 E-mail: lucris@usp.br

3. Advogada. Doutora em Ciências. Docente da Universidade Paulista - campus Sorocaba, Sorocaba (SP), Brasil. 0RCID: 0000-0003-3979-6686

E-mail: evelynsantinon@uol.com.br

4. Enfermeira. Doutoranda pelo Programa Interunidades de Doutoramento em Enfermagem da Escola de Enfermagem da Universidade de São Paulo, Ribeirão Preto. Especialista do Laboratório de Obstetrícia da Escola de Artes, Ciências e Humanidades da Universidade de São Paulo, São Paulo (SP), Brasil. ORCID: 0000-0001-7128-1098 E-mail: kelly.pereira@usp.br

5. Enfermeira. Doutoranda pelo Programa Interunidades de Doutoramento em Enfermagem da Escola de Enfermagem da Universidade de São Paulo (USP) e da Escola de Enfermagem de Ribeirão Preto da USP. Docente da Escola de Artes, Ciências e Humanidades da USP, São Paulo, Brasil. ORCID: 0000-0003-3383-1540 E-mail: fernandamarcal@usp.br

6. Enfermeira. Doutoranda pelo Programa de Ciências de Enfermagem da Universidade do Porto no Instituto de Ciências Biomédicas Abel Salazar - ICBAS/U.PORTO. Especialista do Laboratório do Curso de Obstetrícia da Escola de Artes, Ciências e Humanidades da USP, São Paulo (SP), Brasil. ORCID: 0000-0001-9171-0865 E-mail: joyce@usp.br 


\section{INTRODUÇÃO}

D entre as definições de adolescência, algumas estão associadas aos parâmetros etários ${ }^{1}$ segundo a qual é a fase de desenvolvimento correspondente à faixa etária de 10 a 19 anos de idade.

Os dados preliminares para o ano de 2015, do Departamento de Informática do Sistema Único de Saúde (DATASUS) através do Sistema de Informações de Nascidos Vivos, revelam que os nascimentos com idade materna entre 10 e 19 anos representaram $18,1 \%$ do total de nascidos vivos no Brasil, com maior incidência nas regiões Nordeste e Sudeste ${ }^{2}$.

A gravidez na adolescência é um fenômeno com repercussões social, cultural, legal, psicoemocional e corporal, sendo necessário um olhar cuidadoso na perspectiva da atenção à saúde materno-fetal ${ }^{3,4}$.

A gravidez na adolescência não é, necessariamente, um problema. Mas, a gestante, especialmente na adolescência, demanda atenção com abordagem interdisciplinar e suporte intersetorial para que a vivência da gravidez tenha possíveis efeitos desfavoráveis minimizados ${ }^{3,4}$.

Nessa perspectiva, prioriza-se como cuidado básico de saúde a promoção da maternidade segura para adolescentes, programas de cuidado à saúde especialmente elaborados para esse público, uma vez que, embora suas necessidades clínicas sejam as idênticas a de outras mulheres, precisam de maior suporte para desenvolver habilidades para o cuidado de saúde durante o ciclo gravídico puerperal ${ }^{5}$.

Evidencia-se aqui a importância da assistência pré-natal, que compreende um conjunto de atividades visando a promoção da saúde da mulher grávida e do concepto, com identificação de riscos para ambos, visando a assistência oportuna e adequada, com acolhimento dessa mulher desde o início da gravidez, assegurando o bem-estar materno e fetal ${ }^{6}$.

O profissional de saúde é peça fundamental para a estruturação e/ou para o fortalecimento de redes de apoio e suporte social. Esses profissionais devem estar aptos a assistir a adolescente que vivencia a gravidez em todas as dimensões do cuidado, com atuação que enfatize abordagem integral à saúde, da promoção da saúde e da articulação dos setores sociais para o cuidado da adolescente ${ }^{3}$.

A atuação direta e sistemática junto às adolescentes, desde antes da concepção até os cuidados com seu filho, traduz que a inserção da (o) enfermeira (o) na saúde das adolescentes é uma forma de oportunizar os conhecimentos para que sejam construídos e/ou reformulados acerca da vivência nesta fase ${ }^{7}$.

Frente ao exposto, a pergunta de pesquisa deste estudo foi: Como o profissional de saúde, nomeadamente, a (o) enfermeira (o) atua na atenção primária à adolescente grávida?

Este estudo se justifica por entender que a gravidez na adolescência consiste em um processo de vulnerabilidade ao conhecer a experiência da (o) enfermeira (o) que atua na atenção primária de saúde (APS) poderá contribuir para o melhor acolhimento. Portanto, essa pesquisa teve como objetivo conhecer as experiências de enfermeiras (os) da atenção primária de saúde no atendimento pré-natal de adolescentes, na região de São Miguel Paulista, zona leste de São Paulo (SP).

\section{MÉTODO}

Para investigar a atuação das (os) enfermeiras (os) no pré-natal das adolescentes, utilizou-se uma abordagem qualitativa, pois acredita-se que a mesma pode auxiliar a compreender a experiência profissional junto às gestantes adolescentes.

0 estudo foi realizado em 14 Unidades Básicas de Saúde (UBS) localizadas na região sob a responsabilidade da Coordenadoria Regional de Saúde Leste composta pelas subprefeituras de Cidade Tiradentes, Ermelino Matarazzo, Guaianases, Itaim Paulista, Itaquera, São Mateus e São Miguel Paulista ${ }^{8}$.

Nas Unidades Básicas de Saúde (UBSs) que compuseram o cenário do estudo funcionam Unidades Básicas de Saúde Tradicionais (UBST), Unidades Mistas (UM) e Unidades Básicas Estratégia de Saúde da Família (UBESF), sendo que em todas essas, 
independentemente da modalidade de atenção à saúde, a assistência pré-natal é norteada pelo protocolo "Rede de Proteção à Mãe Paulistana/Rede Cegonha"9,10.

A Rede de Proteção à Mãe Paulistana foi instituída no município de São Paulo em 2006, sendo vinculada às diretrizes e atualizações da Rede Cegonha, programa de abrangência nacional, cinco anos mais tarde.

Para coleta de dados preencheu-se questionário de perfil sociodemográfico e educacional para caracterização dos sujeitos de pesquisa e realizou-se entrevistas semiestruturadas com dezenove enfermeiros que prestavam assistência pré-natal nestes serviços no segundo semestre de 2012.

Os critérios de seleção dos sujeitos foram: atuar na assistência pré-natal, enquanto integrantes da equipe de serviços de APS e, manifestar aceitação voluntária para participar no estudo.

Como técnica de análise de dados qualitativos, optou-se pela Análise de Conteúdo Temática de Bardin ${ }^{11}$. Para armazenagem e gerenciamento dos dados do perfil sócio demográfico e educacional foi elaborada uma planilha eletrônica empregando o programa Microsoft Excel®, o banco de dados foi transportado para o programa StatisticalPackage for the Social Science (SPSS), versão 17.0, para proceder à análise descritiva utilizando frequências absolutas e percentuais.

A pesquisa pautou-se nas diretrizes e normas no466/12 do Conselho Nacional de Saúde, sendo aprovado pelo Comitê de Ética em Pesquisa da Secretaria Municipal de Saúde de São Paulo, sob o parecer $\mathrm{n}^{\mathrm{o}}$ 104/11. Todos os participantes aceitaram voluntariamente aderir à pesquisa e assinaram o Termo de Consentimento Livre e Esclarecido.

\section{RESULTADOS}

Foram entrevistados 19 profissionais, predominantemente do sexo feminino $(89,5 \%)$. Tais profissionais tinham faixa etária entre 29 e 57 anos, sendo 63,2\% deles maiores que 35 anos. Em relação ao estado civil, 57,9\% eram casados e 73,7\% tinham 1 ou mais filhos.
A formação universitária ocorreu majoritariamente em instituições privadas da região metropolitana de São Paulo (94,3\%). No que se refere ao tempo de formação, 21,1\% tinham até 5 anos; $52,6 \%$ entre 6 e 15 anos e 26,3\% tinham 16 anos ou mais de graduação.

Em relação à pós-graduação lato sensu, apenas 15,8\% não haviam cursado. Os cursos realizados eram de áreas afins à atenção básica (71,4\%) como: obstetrícia, saúde pública, saúde da família, saúde coletiva e acupuntura; 23,8\% dos cursos eram afins à área hospitalar: administração hospitalar, cardiologia, urgência e emergência, médicocirúrgica e unidade de terapia intensiva e, $4,7 \%$ voltadas ao ensino, docência. Em se tratando de pós-graduação stricto sensu, apenas um profissional cursou mestrado em Atendimento a Pronto Socorro.

Segundo os anos de atuação na assistência ao pré-natal: 31,6\% tinham até 6 anos; 47,4\% tinham de 6 a 10 anos e 21\% tinham de 11 a 18 anos.

Nas entrevistas realizadas com os enfermeiros emergiu a categoria: Cuidado pré-natal das adolescentes com três subcategorias (Figura 1).

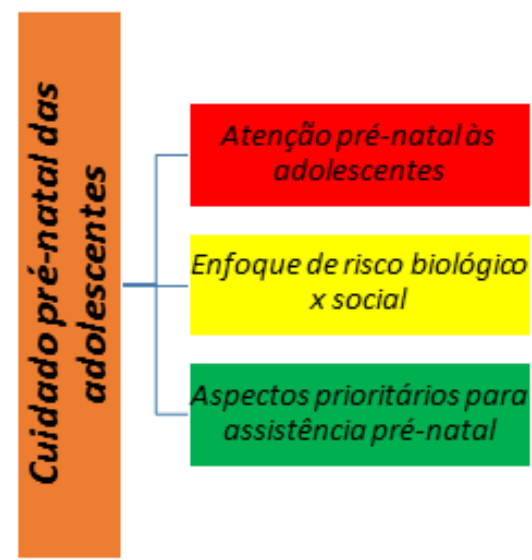

Figura 1. Categoria e subcategorias. Coordenadoria Regional de Saúde Leste Cidade de São Paulo, 2013

\section{Atenção pré-natal às adolescentes}

As mulheres com suspeita de gravidez que chegam ao serviço são acolhidas por um profissional de enfermagem que fará os encaminhamentos e orientações conforme o resultado do exame. Uma vez confirmada a gravidez, inicia-se com o enfermeiro o calendário de consultas da Rede de Proteção à 
Mãe Paulistana/Rede Cegonha, como é evidenciado na fala:

Na primeira consulta: ela faz o pregnosticon [...]. Eu já abro o SisPrenatal, peço os exames de rotina, faço as orientações necessárias, receito o ácido fólico e peço para ela marcar a próxima consulta. Faço o pedido para o cartão SPtrans, temos a agenda da gestante que vem com todas as orientações [...]. É assim uma consulta bem rica, por que praticamente o tempo que nós temos é de 20 minutos para atender cada gestante, mas acabamos levando muito mais, 40, 50 minutos. É porque o SisPrenatal é um formulário com muitos dados, não é simplesmente preencher os dados da gestante, verificar peso, altura, pressão, tem a parte da orientação (Enfermeira 12).

0 atendimento intercalado entre médico e enfermeira, preconizado pelo protocolo da Rede de Proteção à Mãe Paulistana/Rede Cegonha, acontece apenas nas UBESF, já nas UBS tradicionais, as enfermeiras atendem as gestantes somente na primeira e/ou última consulta de pré-natal, ou ainda, em algum encaixe ou imprevisto:

A nossa unidade é tradicional, então a gestante recebe o acolhimento, que é conosco. A consulta fica com o obstetra mensalmente. Então, normalmente a gestante passa na primeira e última consulta de pré-natal com o enfermeiro. Embora a qualquer momento, que ela tenha qualquer dúvida o enfermeiro está aqui a disposição para poder elucidar essas dúvidas ou mesmo encaminhar para que dê solução ao problema relatado (Enfermeira 1).

Foi perguntado às enfermeiras, como estava organizada a assistência à adolescente nos serviços:

A gente foca bastante a gestante, mas não tem nada específico para as adolescentes não (Enfermeira 11).

Verificou-se que não há diferença. Todas as adolescentes gestantes são tratadas da mesma forma que aquelas em outras faixas etárias, mesmo quando os sujeitos do estudo afirmavam que $o$ atendimento às adolescentes era "diferencial", estavam se referindo apenas à escuta mais atenciosa: Para o pré-natal das adolescentes, o diferencial é o cuidado maior, mais orientação, principalmente porque a maioria vem sem acompanhante, sem a mãe, um adulto responsável, então é a gente que tem que dar, a gente tem que orientar (Enfermeira 4).

A escuta qualificada do atendimento "diferencial" preocupava-se principalmente com determinantes sociais e de vulnerabilidade, evidenciados pelas falas:

(...) no acolhimento a primeira coisa que fazemos é estudar qual a situação da adolescente se ela é de risco, se são filhas de pais separados, se são usuárias de drogas, então você tem que fazer toda uma anamnese, um histórico dela (Enfermeira 14).

A gente faz o teste, se positivo, já dá prosseguimento no atendimento, caso não dê positivo ela é orientada para participar dos grupos de adolescentes, que são grupos educativos existentes no posto onde ela vai ter conhecimento da problemática que envolve a gravidez na adolescência (Enfermeira 1).

\section{Enfoque de risco biológico versus risco social}

As adolescentes menores de 16 anos são encaminhadas ao pré-natal de alto risco, quando preciso, porém, elas devem ainda, continuar o atendimento concomitante na unidade de saúde de referência. No entanto, não são atendidas pelas enfermeiras, somente pelos médicos:

(...) uma adolescente menor de 16 anos, a gente também não acompanha, ela é acompanhada no pré natal de risco, e com o médico da unidade. 0 enfermeiro só participa de orientações, mas ele não atende em consulta pré natal, por ser considerado um pré-natal de risco, adolescentes menores de 16 anos (Enfermeira 9).

Ao longo das entrevistas, poucas enfermeiras comentaram especificamente sobre quais os critérios que classificavam a adolescente como uma gestante de risco e por isso encaminhar ao pré-natal especializado, entretanto, houve enfermeira que considerou o risco gestacional em sua fala:

Pela precocidade ela não atingiu a plenitude da maturidade e de repente o organismo dela vai ter que funcionar como se já tivesse atingido, isso impõe algumas alterações. Risco de desenvolver uma hipertensão específica da gestação, uma diabetes gestacional, enfim diversos fatores que poderão desencadear, a gente procura estar alertando (Enfermeira 1).

0 risco social também fez parte das falas das enfermeiras:

Não se gera um filho, se gera problema social (Enfermeira 5).

Aqui é uma área de risco social também, tem um grande número de evasão escolar, alto número de usuários de droga, e tem muito traficante também, o que acontece? Às vezes, elas veem nessas pessoas, uma chance delas escaparem, porque às vezes ela tá lá dentro de casa, moram num cômodo ou dois, com o pai, a mãe e dez irmãos, todo mundo passando fome. Aí vem um cara, supostamente um bandido que vai ter dinheiro, vai tirar ela daquela situação, qual o jeito dela fazer isso? Engravidando. Então, assim a gente vê que é uma ilusão que elas têm de uma expectativa melhor de vida, elas não param para pensar que vai ser mais uma pessoa para sustentar, que vai ficar mais um dentro da casa da mãe (Enfermeira 13). 
Aspectos prioritários na assistência à adolescente grávida

Quando questionadas sobre o que consideravam como prioritário para a assistência pré-natal das adolescentes, as enfermeiras elencaram diversos elementos, como: repetir várias vezes as orientações para gravarem; manter diálogo aberto e sem julgamentos para apoiar, acolher e criar vínculo; ouvir com empatia tudo que têm a contar mesmo que não se relacione à gestação.

Os profissionais reconhecem a importância de suporte para a adolescente em todo o ciclo gravídico-puerperal. Pode-se exemplificar essa constatação por meio da seguinte fala:

Eu considero o apoio, é o apoio psicológico, porque eu acho que na adolescência um grande problema é a questão psicológica, elas não têm muita noção do que é a gestação, enquanto elas estão grávidas, elas ainda estão achando que é uma brincadeira; acho que é muito importante ter esse acompanhamento e a atenção, principalmente no puerpério, por que depois no puerpério há um grande risco dessa adolescente rejeitar essa criança (Enfermeira 13).

Uma outra prioridade é a assistência diferenciada, focada para as adolescentes e com vistas ao estabelecimento de vínculo e adesão ao pré-natal. A demanda é diferente e os enfermeiros precisam adaptar-se a isso:

A gente deveria manter um olhar diferenciado para o adolescente, ou ter uma demanda livre, que ela pudesse ter essa condição de vir na hora que ela pudesse, até na questão do grupo, poderia ter um grupo diferenciado, porque assim, todas são gestante, a gente não separa, então, eu acho que poderíamos pensar em uma assistência diferenciada (Enfermeira 5).

$O$ acolher é primeira coisa que não pode faltar. Você precisa conhecer a família dessa adolescente, em que contexto ela está inserida, porque a partir da hora que você conhecer a realidade dela fica mais fácil acompanhar e até mesmo se houver necessidade, a gente vai abordar a família, principalmente a mãe (Enfermeira 18).

0 modelo de atenção à saúde com visão reducionista, curativista e centrado nos aspectos biológicos permeou alguns discursos:

(...) e depois assim a gente vai fechando o cerco com as questões ginecológicas, de secreções, a procura de streptococos B. Prioridade é ver a situação do feto, se o coração tá batendo, se não tá, ultrassom, se tá formado, se não tá, e depois a gente começa a ver a parte odontológica dela e por aí vai (Enfermeira 05).

De maneira geral, as enfermeiras não conseguiram traçar uma única prioridade e alegaram que toda a assistência é importante para o atendimento à adolescente grávida:

Eu acho que tudo. Por que eu não acho que tenha uma coisa mais prioritária que outra; falar sobre amamentação, falar sobre um método (...) não tem uma coisa prioritária. E específico para a adolescente é o trabalho psicológico depois que nascer, porque a gravidez acho que é de menos, o pior é depois que nascer (Enfermeira 8).

\section{DISCUSSÃo}

Na realização do pré-natal, a enfermeira e os demais profissionais podem desenvolver atividades educativas, captação precoce de gestantes, estímulo ao parto normal, solicitação de exames, avaliação do estado nutricional, prevenção e tratamento, classificação de risco, realização de anamnese, exame físico, entre outros ${ }^{12}$.

0 que pode-se constatar com a entrevista referente a primeira consulta da gestante adolescente é o bombardeio de informações e questionamentos, possível medo, pode ser um grande e talvez assustador inquérito, com solicitações de exames, prescrições de vitaminas, solicitação de transportes, para além da constatação da gravidez que a adolescente vivenciará um processo de internalização.

A consulta de pré-natal, de acordo com Ministério da Saúde (MS), constitui-se como um conjunto de procedimentos clínicos e educativos com o intuito de promover a saúde e identificar precocemente os problemas que possam resultar em risco para a saúde da gestante e o concepto ${ }^{12,13}$.

Essa consulta pode ser realizada pela obstetriz e/ou enfermeira obstétrica e/ou médico obstetra, e também pelo enfermeiro ou médico de família, é composta de ações simples, onde o profissional presta assistência de promoção e prevenção à saúde da mulher e do recém-nascido.

Quando pensa-se na questão do acolhimento, pode-se observar uma série de dificuldades, não só no próprio exercício profissional para com a gestante, mas no entendimento que se tem do significado do termo "acolhimento".

Para o MS, acolher é aceitar, dar ouvidos, dar crédito agasalhar, receber, atender, admitir, ou seja, o acolhimento é uma 
ação de aproximação, um "estar com" e um "estar perto de", uma atitude de inclusão14,15.

Todo lugar onde ocorre um encontro enquanto trabalho de saúde, gera uma relação profissional/usuário, onde produz-se relações de escuta e responsabilização, do qual se constitui vínculos e compromissos em projetos de intervenção ${ }^{16}$.

0 acolhimento nas práticas de produção de saúde está presente em todas as relações e encontros com os pacientes, mesmo quando pouco cuidamos dele ${ }^{14}$. Entretanto, no estudo aqui apresentado parece ter ficado difícil verificar acolhimento em práticas cotidianas. Uma vez que observou-se na relação entre profissional/gestante, um acolhimento aquém ou pouco pleno ao proposto pelo MS nas falas das enfermeiras entrevistadas. Nessa observação, o acolhimento à gestante adolescente, que deveria ser singular e específico às atribuições da sua idade, pode se agravar porque a adolescente que ainda não alcançou a maturidade pode começar a ser tratada como uma mulher madura ou em processo de amadurecimento devido a maternidade.

Para entender a situação e prestar o cuidado à gestante, deve-se ter como objetivo, compreender a subjetividade dessa adolescente, perceber as suas dimensões humanas, tratar da saúde de forma integral, englobar o processo do cuidar para promover, manter e/ou recuperar a dignidade e a totalidade humana ${ }^{17}$. 0 cuidado é entendido como ação que vai além de procedimentos técnicos, pois engloba envolvimento e compromisso com o outro e torna-se por isso, uma ação humanizada, que reconhece o usuário como sujeito e participante ativo no processo de produção de saúde ${ }^{7}$.

Foi possível observar que as falas das enfermeiras estavam arraigadas de julgamentos morais e preconceitos, o que muito provavelmente influencia na assistência prestada a essa adolescente ao longo do pré-natal. Um estudo ${ }^{18}$, verificou que entre os enfermeiros entrevistados acerca da assistência pré-natal à adolescente, os juízos de valores estiveram presentes como: "mal nasceram e já estão mantendo relacionamento sexual" ou "essas crianças, porque quinze, quatorze, dezesseis anos, engravidam por descuido mesmo".

Chamar a adolescente de criança, de descuidada, de precoce, pode ser entendido como ofensas e julgamentos. Isso influencia negativamente a assistência profissional, além disso os julgamentos na maioria das vezes já foram reforçados pelos amigos e pelos familiares.

Outros estudos ${ }^{19,20}$ compartilham da mesma teoria, de que a atuação profissional voltada para saúde materna, requer a satisfação da usuária, principalmente em relação aos profissionais, uma conduta de respeito e atenção, sem julgamentos prévios, proporcionando-a uma gestação mais saudável, considerando que esta é uma fase, em que as modificações físicas e psíquicas, a deixam mais vulnerável e fragilizada.

Para tanto, recomenda-se utilizar estratégias, como a escuta aberta, sem julgamentos e sem preconceitos, além do diálogo franco, permitindo à mulher falar de sua intimidade com segurança, expressando suas dúvidas e necessidades, possibilitando assim o estabelecimento do vínculo profissional-cliente ${ }^{19}$.

Sobre o enfoque de risco biológico e risco social, o Ministério da Saúde ${ }^{12}$ publicou um Caderno de Atenção Básica, denominado "Atenção ao pré-natal de baixo risco", que tem como objetivo orientar o atendimento de acordo com as evidências mais atuais, com intuito de realizar uma prática humanizada e integral, garantindo padrão de acesso e qualidade.

Na parte referente ao pré-natal, há uma classificação de risco gestacional, havendo a indicação de fatores de risco que permitem a realização do pré-natal pela equipe de atenção básica; os que podem indicar encaminhamento ao pré-natal de risco e os que indicam encaminhamento à urgência/ emergência obstétrica.

Em relação aos fatores de risco que permitem a realização do pré-natal pela equipe de atenção básica, estão os relacionados às características individuais e às condições sociodemográficas desfavoráveis. Destaca-se a atenção para a idade menor de 15 anos; a situação familiar 
insegura e a não aceitação da gravidez, principalmente em se tratando de adolescente $^{12}$.

Porém, enfatiza que as adolescentes grávidas não possuem maior risco clínico e obstétrico em relação às grávidas de outras faixas etárias, só pelo fato de serem adolescentes. Uma assistência pré-natal adequada é fundamental para garantir o baixo risco. No entanto, é preciso estar atento às gestantes da faixa etária entre 10 e 14 anos, pois apresentam maiores riscos maternofetais. Entretanto, quando elas recebem atenção qualificada, os resultados se aproximam daqueles da população em geral ${ }^{12}$.

Mesmo o MS considerando um fator de risco gestacional a gestação entre adolescentes de 10 a 14 anos (que podem ser minimizados com atenção qualificada), percebe-se um avanço em relação à abordagem realizada. Por exemplo, no manual de pré-natal publicado no ano de 2000 , não fazia nenhuma ressalva a atenção oferecida à adolescente $^{21}$.

Frente a isso, considera-se importante ressaltar, que mesmo o MS trazendo considerações importantes relacionadas à assistência à gestante adolescente, o protocolo em vigor do município de São Paulo, ainda caracteriza a gestação em adolescentes menores de 16 anos como sendo de risco. Consequentemente, as enfermeiras não podem realizar esse atendimento, como verifica-se nas falas dessas profissionais.

Em relação aos riscos de morte materna, $50 \%$ dos óbitos estão relacionados com causas obstétricas diretas, quais são complicações durante a gravidez, parto ou puerpério. Causadas por intervenções, omissões ou tratamento incorreto, essas são prevenidas com um acompanhamento de prénatal adequado, para um maior conhecimento do estado de saúde da gestante e do desenvolvimento da gestação, não excluindo as medidas educativas e de planejamento familiar ${ }^{22}$.

Ao se tratar dos riscos biológicos das adolescentes, não há evidências que comprovem evolução obstétrica desfavorável relacionada com o único fator de pouca idade materna. As situações de risco biológico mais frequentes, entre as adolescentes muito jovens, são a hipertensão gestacional, prematuridade e baixo peso neonatal, que estão relacionadas não só com a idade materna, mas também às condições psicossociais inadequadas, e à qualidade da assistência pré-natal e do parto ${ }^{23}$.

Em outro estudo ${ }^{24}$ que, o pré-natal inadequado contribuiu, significativamente, para o aumento do risco gestacional em adolescentes, como por exemplos, maior prematuridade, maior risco para baixo peso ao nascer, apgar de quinto minuto menor que sete, entre outros.

Atualmente acredita-se que uma assistência de qualidade resolveria ou minimizaria o risco biológico, visto que um dos principais objetivos da atenção pré-natal é exatamente a identificação dos fatores que poderiam colocar o binômio sob um maior risco de um resultado adverso, bem como intervir para evitar iatrogenias ${ }^{25}$.

A situação de desigualdade social, política e econômica encontrada no Brasil tem influência direta na dinâmica familiar e no aumento do número de crianças e adolescentes em situação de risco social e pessoal. Nesse contexto, a gravidez na adolescência tem sido tradicionalmente tratada como um problema de saúde pública, apesar de diferentes estudos atribuírem significações positivas para a vivência da maternidade a partir da perspectiva das adolescentes ${ }^{23,26}$.

Esses riscos não médicos que envolvem a adolescente podem ser: necessidade de ajuda financeira, problemas de moradia, tensão entre os familiares acerca da gravidez não planejada, o medo e a falha em assumir responsabilidades e suas funções de adolescente e de mãe, os hábitos de vida (fumo, álcool, drogas), construir uma nova conformação familiar estável, de se auto estruturarem e criar seus filhos com saúde. E mesmo após a gestação, a necessidade de ajuda financeira e física para o cuidado com o filho permanece, visto que a adolescente normalmente não possui aval do companheiro ou ele também é adolescente e os dois não conseguem assumir as despesas e cuidados totais da criança ${ }^{23,26}$. 
Isso confirma que ao propiciar o cuidado pré-natal (manter vínculo e identificar dificuldades) bem como, acompanhamento puerperal na UBS pode evitar mortes maternas se a assistência for de qualidade. Nesse sentido, fomenta-se a vantagem e a importância das visitas domiciliares no puerpério e da Estratégia de Saúde da Família, implementada em algumas unidades de saúde da cidade de São Paulo.

Na ESF, a família deve ser entendida de forma integral e em seu espaço social e para entender a pessoa deve ser analisado o contexto socioeconômico e cultural, reconhecê-la como sujeito social portadora de autonomia, e corroborar que é na família que ocorrem interações e conflitos que influenciam diretamente na saúde das pessoas. Assim o foco do cuidado, deve estar em ajudar e em capacitar a família, de forma que ela possa atender às necessidades de seus membros, especialmente em relação ao processo saúde-doença, mobilizando recursos, promovendo apoio mútuo $\mathrm{e}$ crescimento ${ }^{27}$.

Sabendo disso, uma crítica sobre a prática das enfermeiras no programa é que essas acreditam estar cuidando da família mesmo quando o seu processo de trabalho não se diferencia daquele adotado na assistência ao indivíduo, ou seja, estão prestando assistência ao indivíduo que têm familiares e não a assistência à família sob o aspecto de unidade de cuidado ${ }^{27}$.

Já um estudo comparativo de Unidade Básica Tradicional e Unidade Básica de Estratégia de Saúde da Família, nos diferentes estratos de exclusão social, mostrou que para os usuários o índice de atenção básica, no geral, a UBESF foi superior à UBS tradicional, enquanto para os profissionais e gestores esse índice não revelou diferenças relevantes ${ }^{28,29}$.

0 período pré-natal é um momento de grande expectativa e de preparação biológica e psicológica para o parto e para a maternidade. A enfermeira, durante o prénatal, busca contribuir para a promoção da saúde do binômio, através de informações e reflexões quanto à experiência da maternidade, as mudanças do corpo, a adoção de práticas para manutenção da saúde e mudanças de hábitos para solucionar problemas ocasionados pela gestação.

Após intensas discussões e reflexões sobre a assistência à mulher no pré-natal, um consenso sobre a maior receptividade das gestantes às estratégias de atenção à saúde reforça a participação efetiva da mulher no pré-natal que possibilita a aquisição de novos conhecimentos, amplia sua percepção corporal para a sua capacidade de gestar, parir e maternar ${ }^{7,30}$.

É interessante pensar que alguns aspectos citados como prioritários, também foram apontados como dificuldades na assistência. Isso possibilita inferir que, apesar da dificuldade que as enfermeiras têm em dar atenção, apoiar, acolher a demanda que há diariamente nessas unidades, posturas como estas tornam-se fundamentais para as adolescentes, as quais sentem-se acolhidas e cuidadas, e proporciona uma maior adesão ao pré-natal, segundo a percepção de algumas enfermeiras.

A assistência à gestante adolescente deve ocorrer através de ações que visam melhorar o acesso dessas mulheres aos serviços de saúde contando com profissionais capacitados continuamente, para atender essas necessidades específicas dessa faixa etária. Nas Unidades de Saúde, é necessário que sejam feitas intervenções educativas multidisciplinares para adolescentes, não de forma fragmentada e que valorize o biológico, mas buscando um cuidado holístico, voltado para as necessidades biopsicossociais $24,27,31$.

Ao valorizar os aspectos relacionais, as gestantes consideram que a atenção deve ser voltada para uma abordagem que as perceba em sua totalidade, enfatizando a necessidade de maior vínculo com os profissionais de saúde. Nesta perspectiva, caminhos podem ser traçados rumo à reformulação do sistema de saúde hegemônico e vigente, de modo a torná-lo, quiçá, mais orientado para o amparo dos diferentes sujeitos que compõem a (complexa) realidade em prol de um cuidado em saúde mais humanizado e solidário ${ }^{32}$.

Uma pesquisa com adolescentes gestantes abordou as especificidades distintas em relação às gestantes adultas, tendo o médico e enfermeiro papéis fundamentais 
numa assistência singular e diferenciada. Esses profissionais como membros da rede de apoio à mulher, precisam compreender as mudanças físicas, emocionais e sociais vividas pela adolescente, assim como os recursos que as mesmas dispõem e quais suas formas de enfrentamento a estas situações. Dessa forma, a atenção pré-natal realizada à adolescente grávida deve ser "diferenciada" e personalizada em virtude das características próprias deste grupo ${ }^{33}$.

A relação entre o profissional de saúde e adolescente deve ser trabalhada, para que haja mudanças nos saberes pré-estabelecidos e de pré-conceitos. Ao fazer isso, os profissionais podem refletir e encontrar uma forma de se comportar em relação à adolescentes, propiciar participação e conceder informações, além de poder oferecer escuta e atenção à adolescente ${ }^{33}$.

A maternidade na adolescência então, não é apenas um ato biológico-reprodutivo, mas um processo social que afeta significativamente as relações entre homem, mulher e membros familiares, definindo novas identidades sociais. Dessa maneira, deve-se ter um olhar focado para além das questões físico biológicas, uma visão holística, centrada no respeito e aceitação da gestante adolescente, para que a ação humanizada possa abranger todos os conceitos ${ }^{34}$.

\section{CONCLUSÃO}

0 estudo permitiu a reflexão acerca do cuidado humanizado e da postura adotada pelas enfermeiras frente ao cuidado pré-natal das adolescentes.

Percebe-se que a gestante adolescente não recebe, em sua totalidade, uma atenção específica e direcionada, que atenda às suas necessidades singulares de sua faixa etária e condição de gestação.

Em relação às características profissionais das enfermeiras que prestam assistência pré-natal, poucas possuíam especialização em áreas afins à APS, na qual, a maioria não era especializada. Fator que pode impactar fortemente a assistência, no que se refere à falta de preparo específico e de qualificação profissional.
Os

profissionais reconheceram fragilidades no atendimento à gestante adolescente e listaram algumas dificuldades, dentre as quais, o despreparo na formação para essa atuação, questões sociodemográficas e culturais e, aquelas relacionadas às políticas de saúde.

É extremamente necessária a implementação de políticas públicas direcionadas às gestantes adolescentes nos serviços, assim como, investir na capacitação/sensibilização do profissional de enfermagem para a assistência à adolescente, promovendo mudanças na estrutura assistencial com equipes interdisciplinares e multiprofissionais, para um cuidado direcionado e eficiente.

Evidencia-se, dessa maneira, que é necessário realizar ações que visem à assistência integral às adolescentes, não apenas no período gravídico-puerperal. Para isso, é fundamental acolhimento, fortalecimento dos laços entre profissionais e usuárias, resolutividade dos profissionais, discussões permanentes e capacitação permanente da equipe.

\section{REFERÊNCIAS}

1. World Health Organization. Pregnant adolescents: delivering on global promises of hope. Geneva: WHO; 2006. 31p.

2. Ministério da Saúde (Brasil). Pesquisa Nacional de Demografia e Saúde da Criança e da Mulher - PNDS 2006: dimensões do processo reprodutivo e da saúde da criança. Brasília: Ministério da Saúde; 2009. 3. Mandú ENT. Adolescência: o cuidar nessa fase do ciclo vital. In: Fernandes RAQ, Narchi NZ, organizadores. Enfermagem e saúde da mulher. Barueri: Manole; 2007. p.190-210.

4. Rosa AJ, Reis AOA, Tanaka ACD’A. Gestações sucessivas na adolescência. Rev Bras Crescimento Desenvolv Hum. [Internet]. 2007 [citado em 30 mar 2017]; 17(1):165-72. Disponível em: http://pepsic.bvsalud.org/scielo.php?script=sci_art text\&pid=S0104-

$12822007000100017 \& \operatorname{lng}=$ pt\&nrm=is\&tlng=pt.

5. World Health Organization, Department of Making Pregnancy Safer. Making pregnancy safer: a newsletter of worldwide activity [Internet]. Geneva: WHO; 2007 [citado em 30 mar 2017]. 8 p. Disponível em:

http://www.who.int/maternal_child_adolescent/do cuments/newsletter/mps_newsletter_issue4.pdf. 6. Secretaria de Estado da Saúde, São Paulo (Estado), Coordenadoria de Planejamento em Saúde, 
Assessoria Técnica em Saúde da Mulher. Atenção à gestante e à puérpera no SUS - SP: manual técnico do pré natal e puerpério. São Paulo: SES/SP; 2010, 234p.

7. Parenti PW, Silva LCFP, Melo CRM, Clapis MJ. Cuidado pré-natal às adolescentes: competências das enfermeiras. Rev Baiana Enferm. [Internet]. 2012 [citado em 30 mar 2017]; 26(12):498-509. Disponível

em:

http://www.portalseer.ufba.br/index.php/enferma gem/article/view/6534.

8. Governo do Estado de São Paulo, São Paulo (Estado). Lei no 14.544, de 14 de setembro de 2011. Autoriza o Poder Executivo a instituir o Programa "Rede de Proteção à Mãe Paulista", e dá outras providências [Internet]. D.O.E-SP., São Paulo, 15 set 2011 [citado em 30 mar 2017]. Disponível em : http://www.legislacao.sp.gov.br/legislacao/dg2802 02.nsf/ae9f9e0701e533aa032572e6006cf5fd/3a56 959eff3d623f8325790d004dad6b?OpenDocument 9. Prefeitura de São Paulo (Município). Decreto $\mathrm{n}$-46.966, de 02 fevereiro de 2006. Regulamenta a Lei no 13.211, de 13 de novembro de 2001, estruturando a Rede de Proteção à Mãe Paulistana para a gestão e execução da rede de serviços de saúde de assistência obstétrica e neonatal no Município de São Paulo [Internet]. São Paulo, Secretaria do Governo Municipal; 2006. Disponível em:

http://www3.prefeitura.sp.gov.br/cadlem/secretar ias/negocios_juridicos/cadlem/integra.asp?alt $=030$ 22006D\%20469660000.

10. Ministério de Saúde (Br). Portaria no 1.459 , de 24 de junho de 2011. Institui, no âmbito do Sistema Único de Saúde - SUS - a Rede Cegonha [Internet]. D.O.U., Brasília, DF, 27 jun 2011 [citado em 30 mar 2017]. Disponível em: http://bvsms.saude.gov.br/bvs/saudelegis/gm/20 11/prt1459_24_06_2011.html.

11. Bardin L. Análise de conteúdo. 7ed. São Paulo: Almedina; 2011. 229p.

12. Ministério da Saúde (Br), Secretaria de Atenção à Saúde, Departamento de Atenção Básica. Atenção ao pré-natal de baixo risco. Brasília, DF: Ministério da Saúde; 2013. 318 p. (Cadernos de Atenção Básica; n. 32)

13. Ministério da Saúde (Br), Secretaria de Políticas de Saúde, Área Técnica da Mulher. Pré-natal e puerpério: atenção humanizada e qualificada. Brasília, DF: Ministério da Saúde; 2006. 163p.

14. Ministério da Saúde (Br), Secretária de Atenção à Saúde, Departamento de Atenção Básica. Acolhimento à demanda espontânea. Brasília, DF: Ministério da Saúde; 2013. 56 p. (Cadernos de Atenção Básica, n.28).

15. Ministério da Saúde (Br), Secretária de Atenção à Saúde, Departamento de Ações Programáticas Estratégicas. Manual de acolhimento e classificação de risco em obstetrícia. Brasília, DF: Ministério da Saúde; 2014.

16. Coutinho LRP, Barbieri AR, Santos MLM. Acolhimento na atenção primária à saúde: revisão integrativa. Saúde Debate [Internet]. 2015 [citado em 30 mar 2017]; 39(105):514-24. Disponível em: http://www.scielo.br/pdf/sdeb/v39n105/0103-

1104-sdeb-39-105-00514.pdf

17. Reis ABF, Silva JLL, Andrade M. Assistência das adolescentes gestantes na estratégia de saúde da família. Informe-se em Promoção da Saúde [Internet]. 2009 [citado em 30 mar 2017]; 5(2):23-5 Disponível

em:

http://www.uff.br/promocaodasaude/adolescentes \%20gestantes8.pdf

18. Santos DR, Maraschin MS, Caldeira S. Percepção dos enfermeiros frente à gravidez na adolescência. Ciênc Cuid Saúde [Internet]. 2007 [citado em 30 mar 2017]; 6(4):479-85 Disponível em: http://periodicos.uem.br/ojs/index.php/CiencCuid Saude/article/view/3684.

19. Anversa ETR, Bastos GAN, Nunes LN, Dal Pizzol TS. Qualidade do processo da assistência pré-natal: unidades básicas de saúde e unidades de Estratégia Saúde da Família em município no Sul do Brasil. Cad Saúde Pública [Internet]. 2012 [citado em 02 mar 2017]; 28(4):789-800. Disponível em: http://dx.doi.org/10.1590/S0102-

311X2012000400018.

20. Guanabens MFG, Gomes AM, Mata ME, Reis ZSN. Gravidez na adolescência: um desafio à promoção da saúde integral do adolescente. Rev Bras Educ Méd. [Internet]. 2012 [citado em 30 mar 2017]; 36(1, Supl2):20-4. Disponível em: http://www.scielo.br/pdf/rbem/v36n1s2/a04v36 n1s2.pdf.

21. Ministério da Saúde (Brasil). Manual técnico de assistência pré-natal. Brasília, DF: Ministério da Saúde; 2000. 66p.

22. Silva JLP, Surita FGC. Gravidez na adolescência: situação atual. Rev Bras Ginecol Obstet. [Internet]. 2012 [citado em 30 mar 2017]; 34(8):347-50. Disponível em: http://dx.doi.org/10.1590/S010072032012000800001.

23. Oliveira M, Coimbra V, Pereira A. Complicações na gravidez adolescente em situação de risco social. Rev E-Psi. [Internet]. 2015 [citado em 30 mar 2017]; 5(2):35-50. Disponível em: https://revistaepsi.com/wpcontent/uploads/artigos/2015/Ano5-Volume2Artigo3.pdf.

24. Nader PRA, Cosme LA. Parto prematuro de adolescentes: influência de fatores sociodemográficos e reprodutivos, Espírito Santo, 2007. Esc Anna Nery Rev Enferm. [Internet]. 2010 [citado em 30 mar 2017]; 14(2):338-45. Disponível em: http://dx.doi.org/10.1590/S141481452010000200018 
25. Enkin M, Keirse M, Neilson J, Crowther C, Duley L, Hodnett E, et al. Guide to effective care in pregnancy and childbirth. 3ed. Oxford: Oxford University Press; 2000.

26. Araújo RLD, Rodrigues ES, Oliveira GG, Sousa KMO. Gravidez na adolescência: consequências centralizadas para a mulher. Temas Saúde [Internet]. 2016 [citado em 30 mar 2017]; 16(2):567-87. Disponível em: http://temasemsaude.com/wpcontent/uploads/2016/08/16231.pdf

27. Deprá AS, Heck RM, Thum M, Ceolin T, Vanini M, Lopes CV, et al. Gravidez de adolescentes na unidade de saúde da família. Rev Enferm Cent-Oest Min. 2011; (1):59-69.

28. Elias PE, Ferreira CW, Alves MCGóis, Cohn A, Kishima V, Escrivão Jr A, et al. Atenção básica em saúde: comparação entre PSF e UBS por estrato de exclusão social no município de São Paulo. Ciênc saúde coletiva. [Internet]. 2006 [citado em 02 mar 2017]; 11(3):633-41. Disponível em: http://dx.doi.org/10.1590/S1413-

81232006000300012

29. Van Stralen CJ, Belisário SA, Van Stralen TBS, Lima ÂMD, Massote AW, Oliveira CL. Percepção dos usuários e profissionais de saúde sobre atenção básica: comparação entre unidades com e sem saúde da família na Região Centro-Oeste do Brasil. Cad Saúde Pública [Internet]. 2008 [citado em 02 mar 2017]; 24( Suppl 1 ):s148-s58. Disponível em: http://dx.doi.org/10.1590/S0102-

311X2008001300019.

30. Teixeira IR, Amaral RMS, Magalhães SR. Assistência de enfermagem ao pré natal: reflexão sobre a atuação do enfermeiro para o processo educativo na saúde gestacional da mulher. e-Scientia [Internet]. 2010 [citado em 30 mar 2017]; 3(2):26$31 . \quad$ Disponível em: http://revistas.unibh.br/index.php/dcbas/article/v iew/166.

31. Federação Brasileira das Associações de Ginecologia e Obstetrícia. Manual de ginecologia infanto juvenil. São Paulo: FEBRASGO; 2014. 182p.

32. Barbaro MC, Lettiere A, Nakano AMS. Assistência pré-natal à adolescente e os atributos da Atenção Primária à Saúde. Rev Latinoam Enferm. [Internet]. 2014 [citado em 02 mar 2017]; 22(1):108-14. Disponível em: http://dx.doi.org/10.1590/01041169.3035.2390.

33. Buendgens BB, Zampieri MFM. A adolescente grávida na percepção de médicos e enfermeiros da atenção básica. Esc Anna Nery Rev Enferm. [Internet]. 2012 [citado em 02 mar 2017]; 16(1):64$72 . \quad$ Disponível

http://dx.doi.org/10.1590/S141481452012000100009.

34. Moreira TMM, Viana DS, Queiroz MVO, Jorge MSB. Conflitos vivenciados pelas adolescentes com a descoberta da gravidez. Rev Esc Enferm USP. [Internet]. 2008 [citado em 02 mar 2017]; 42(2): 312-20. Disponível em: http://dx.doi.org/10.1590/S008062342008000200015

\section{CONTRIBUIÇÕES}

Todas autoras tiveram iguais contribuições.

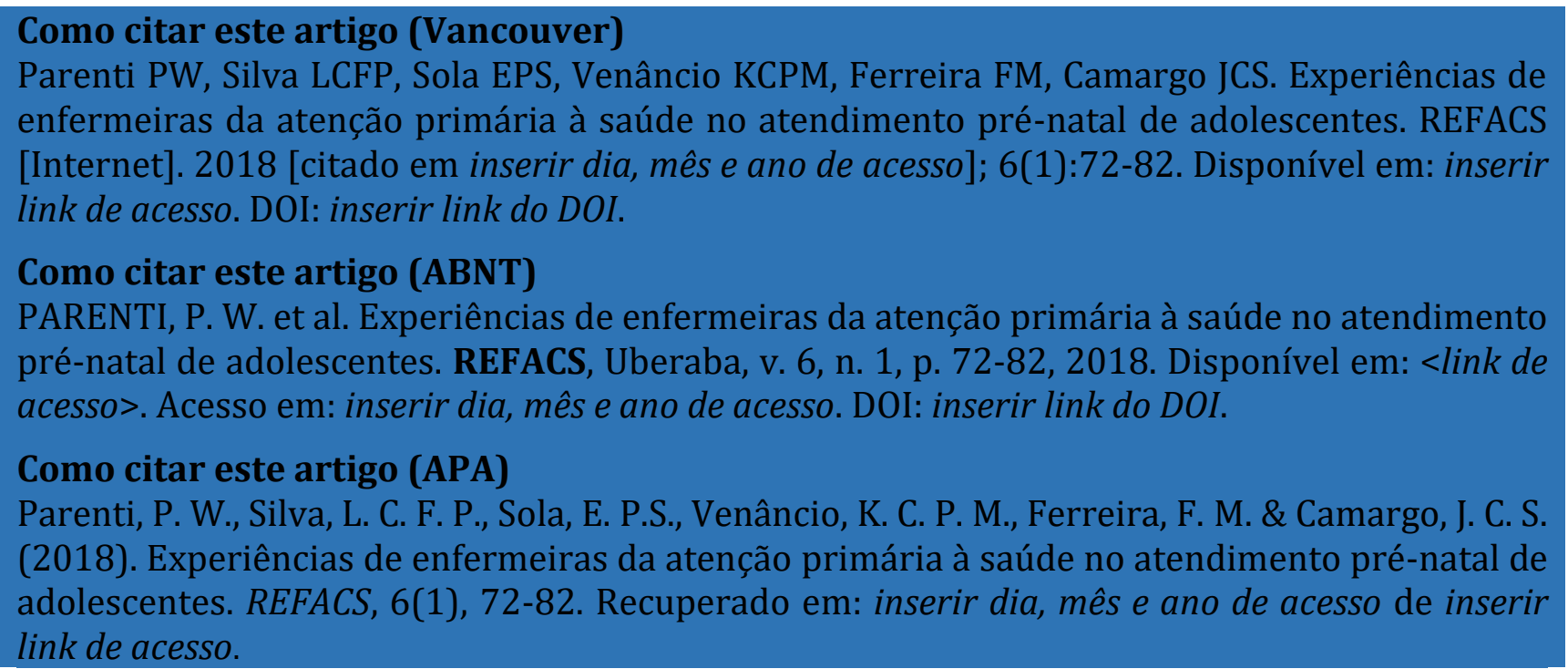

